\title{
The neural correlates of placebo effects: a disruption account
}

\author{
Matthew D. Lieberman, * Johanna M. Jarcho, Steve Berman, Bruce D. Naliboff, \\ Brandall Y. Suyenobu, Mark Mandelkern, and Emeran A. Mayer
}

University of California, Los Angeles, CA 90095-1563, USA

Received 3 November 2003; revised 15 January 2004; accepted 15 January 2004

\begin{abstract}
The neurocognitive pathways by which placebo effects operate are poorly understood. Positron emission tomography (PET) imaging was used to assess the brain response of patients with chronic abdominal pain (irritable bowel syndrome; IBS) to induced intestinal discomfort both before and after a 3 -week placebo regimen. A daily symptom diary was used to measure symptom improvement. Increases in right ventrolateral prefrontal cortex (RVLPFC) activity from pre- to postplacebo predicted self-reported symptom improvement, and this relationship was mediated by changes in dorsal anterior cingulate (dACC), typically associated with pain unpleasantness. These results are consistent with disruption theory [Lieberman, M.D., 2003. Reflective and reflexive judgment processes: a social cognitive neuroscience approach. In: Forgas, J.P., Williams, K.R., von Hippel, W. (Eds.), Social Judgments: Explicit and Implicit Processes. Cambridge Univ. Press, New York, pp. 44-67], which proposes that activation of prefrontal regions associated with thinking about negative affect can diminish dACC and amygdala reactivity to negative affect stimuli. This is the first study to identify a neural pathway from a region of the brain associated with placebos and affective thought to a region closely linked to the placebo-related outcome of diminished pain unpleasantness.

(C) 2004 Elsevier Inc. All rights reserved.
\end{abstract}

Keywords: Neural correlates; Placebo effects; Disruption

\section{Introduction}

Placebo effects and the power of belief over physical outcomes in the body have a history as long as medicine itself. Only recently, however, have the neural bases of these psychophysiological phenomena begun to be examined. Current research has shown that placebos can produce changes in brain activity similar to the pharmacological agents they are replacing. For instance, placebos administered in the treatment of depression have been shown to alter brain activity in much the same way as fluoxetine, an effective antidepressant medication (Mayberg et al., 2002). Such findings suggest that the final result of placebo administration may mimic the effects of active agents; however, the mediating neurocognitive

* Corresponding author. Department of Psychology, University of California, Los Angeles, Franz Hall, 405 Hilgard Avenue, Los Angeles, CA 90095-1563. Fax: +1-310-206-5895.

E-mail address: lieber@ucla.edu (M.D. Lieberman).

Available online on ScienceDirect (www.sciencedirect.com.) process by which ingesting a placebo and placebo-related beliefs lead to these final neural results has not yet been examined. In other words, it is unknown what placebo-specific neural activity sets in motion the subsequent symptom-specific changes in brain activity. In a positron emission tomography (PET) study of placebo effects on visceral pain, we investigated a possible mediating mechanism derived from disruption theory (Eisenberger et al., 2003; Lieberman, 2003; Lieberman et al., 2003), which suggests that thinking about affective processes has the unintended effect of diminishing the reactivity of brain regions involved in the automatic generation of negative affect.

Neuroimaging research on placebo effects has focused primarily, though not exclusively, on the neural outcome of placebo effects - identifying changes in the brain regions that most directly relate to subjective changes in symptoms, and comparing these changes with those stemming from active pharmacological agents (de la Fuente-Fernandez et al., 2001; Leuchter et al., 2002; Mayberg et al., 2002). This research has been critical in demonstrating that top-down, belief-related placebo effects modulate the activity of brain regions ordinarily affected by other treatments that presumably operate through bottom-up mechanisms that are not beliefrelated. Such work speaks to the longstanding concern regarding whether placebo effects and other expectancy effects are real, experimental artifacts, or self-presentational effects (Hrobjartsson and Gotzsche, 2001). It is not clear from previous neuroimaging studies, however, whether the neural regions that have been identified are the neural correlates of the placebo effect or the neural correlates of the placebo effect's downstream consequences on symptom outcomes. Though a drug and a placebo may both affect brain region $X$, the drug may do so directly, whereas placebo effects are typically mediated by placebo-induced thoughts. ${ }^{1}$

In previous studies, right prefrontal cortex, anterior cingulate and striatal dopamine have each been implicated in placebo effects, but it is not clear that these are all plausible neurocognitive candidates for transforming placebo-induced thoughts into altered experience. Petrovic et al. (2002) recently compared placeboinduced analgesia to opioid-induced analgesia and found that whereas both placebos and induced opioids led to similar changes

\footnotetext{
1 There are also placebo effects that stem from classical conditioning (Amanzio and Benedetti, 1999); however, the neuroimaging research that has been done to date has not focused on this type of effect. Our comments are thus limited to placebo effects in which prior conditioning to an active agent has not occurred.
} 
in the rostral anterior cingulate and brainstem, only the placebo led to increased activity in right prefrontal cortex $(x=30, y=30, z=$ $-6)$. Though Petrovic et al. suggested this region may be involved in cognitive regulation of pain, a neurocognitive model of this process has yet to emerge. We begin with a brief review of the neuroanatomy of pain and then describe how disruption theory (Eisenberger et al., 2003; Lieberman, 2003), a model of the cognitive regulation of affect, may be applied in the context of pain and placebo effects.

\section{Pain unpleasantness and anterior cingulate}

The experience of pain can be divided into sensory and affective aspects, corresponding to the sensory intensity and the unpleasantness of pain, respectively (Melzack and Casey, 1968; Price, 2000). In recent years, neuroimaging work has suggested that these two aspects of pain are neurally dissociable as well. Whereas the sensory aspects of pain, including its intensity and location, are represented primarily in somatosensory cortex and the insula (Peyron et al., 1999; cf. Coghill et al., 1999), pain unpleasantness is represented in the dorsal anterior cingulate (dACC; Peyron et al., 2000; Rainville et al., 1997; Tolle et al., 1999). Additionally, patients who have had their anterior cingulate surgically removed report that they are still able to feel the intensity of pain, but are no longer bothered by it (Foltz and Lowell, 1962). In contrast, a patient who had his somatosensory cortex removed could still report pain distress despite difficulties in reporting on sensory aspects of the pain (Ploner et al., 1999).

We take the established relationship between dACC's response to painful stimulation and experienced pain unpleasantness as a starting point for our investigation. We assume that changes in the dACC represent one possible end result of a placebo's neurocognitive effects in the domain of pain analgesia. ${ }^{2}$ Using dACC changes as a neural anchor or endpoint indicative of changes in the unpleasant experience of pain, functional connectivity analyses (Lieberman et al., in press; Poldrack et al., 2001) can be used to determine which other brain regions may be modulating the reactivity of dACC and the subjective reports of pain. Functional connectivity analyses are entirely correlational, and thus cannot determine causal links directly. Such links can be inferred with greater confidence to the extent that a strong theoretical rationale and preestablished neural pathways suggest a route by which the effects may be achieved. Disruption theory (Lieberman, 2003) posits that right ventrolateral prefrontal cortex (RVLPFC), the same region identified by Petrovic et al. (2002) in the cognitive regulation of pain, is well-suited to transform placebo-induced beliefs into at least some of its known subjective and neural outcomes.

\section{Disruption theory}

Disruption theory (Lieberman, 2003) proposes that the reflective conscious processes that are engaged in response to automatic

\footnotetext{
2 It is possible that dACC changes represent a combination of changes to pain unpleasantness, pain intensity, and attention to pain (see Eisenberger and Lieberman, in press, for a review). Subjectively, each of these could contribute to placebo effects, so in this study we have not tried to differentiate between these different contributors. We have focused on pain unpleasantness because this has been most directly and frequently associated with dACC in the context of pain.
}

negative affective processes tend to inhibit or 'disrupt' the very same negative affective processes by virtue of a hardwired feedback mechanism. Reflective, nonautomatic, conscious processes, marked by intentionality, effort, awareness, and propositional structure, are typically used sparingly because they are fragile and limited, despite their flexibility (Wegner and Bargh, 1998). For example, keeping one phone number in mind is relatively easy, yet keeping two in mind simultaneously is nearly impossible. Thus, people tend to be 'cognitive misers' (Fiske and Taylor, 1991), using as little of this limited resource as possible so that it will be available when it is really needed. If the cognitive miser view is correct, then it would be advantageous to have an alarm system that signals when reflective conscious processes are really needed. Previous theories (LeDoux, 1996; Schwarz and Bless, 1991) suggest that negative affect serves precisely this function. Disruption theory hypothesizes that once negative affect has sounded the alarm and set reflective processing in motion to respond to the current situation, it is adaptive to have the alarm turned off or at least muted a bit. If the negative affect alarm continues to sound at the same 'volume', it is likely that some reflective processing resources will be consumed by attending to the alarm, rather than operating on the environment to respond to whatever caused the negative affect. Consider the extreme case of panic when the sense of anxiety and alarm is so overwhelming that the individual is essentially paralyzed, unable to respond to real threats in the environment effectively.

Disruption theory suggests that primates evolved a hardwired feedback mechanism such that the activation of brain regions associated with reflective processing responses to negative affect will disrupt, at least to some extent, the activity of brain regions that generate the negative affect alarm. Such a disruption mechanism would keep the distracting effect of an automatic affect alarm to a minimum once the alarm has been initially registered, promoting a more efficient and focused response to the event(s) activating the alarm.

Disruption theory posits RVLPFC as a focal point because it is involved both in reflective conscious responses to negative affect and the disruption of activity in the neural sources of negative affect in the amygdala and anterior cingulate. RVLPFC has been implicated in numerous studies of inhibition generally (Aron et al., 2003; Garavan et al., 1999; Iversen and Mishkin, 1970; Konishi et al., 1999), and overcoming negative affect more specifically (Eisenberger et al., 2003; Hariri et al., 2000; Monchi et al., 2001; Small et al., 2001).

A number of studies have also implicated VLPFC in negative affective processes (Eugene et al., 2003; Gottfried et al., 2002; Markowitsch et al., 2003); however, disruption theory suggests that this region supports the labeling, interpretation, and thought about negative affect rather than the generation or experience of negative affect itself. Five studies have shown greater VLPFC activation when individuals were required to make explicit judgments of affect present in pictures compared to when the affective valence of the pictures was incidental to the judgment dimension (Cunningham et al., 2003; Gorno-Tempini et al., 2001; Gur et al., 2002; Nakamura et al., 1999; Narumoto et al., 2000). One of these studies found VLPFC to be more active for negative judgments than positive judgments (Cunningham et al., 2003). A sixth study found VLPFC activity when individuals were required to make propositional appraisals of affective stimuli (Schaefer et al., 2003). There are other areas that have also been implicated in reflective processing of affect including dorsomedial PFC and ACC (Crosson 
et al., 1999; Teasdale et al., 1999); however, these regions have not been implicated in most studies of inhibition. It is the joint involvement in inhibition and reflective emotional processing that is central to disruption theory.

RVLPFC also has the neuroanatomical connections necessary to inhibit negative affective processes. RVLPFC comprises Brodmann area (BA) 47, as well as BA44 and BA45 in the human, and is considered homologous with primate area 12 in lateral orbitofrontal cortex (LOFC; Ongur and Price, 2000). LOFC has dense projections to the amygdala and dACC (Carmichael and Price, 1995; Cavada et al., 2000; Vogt and Pandya, 1987). Most specific to a disruption account of placebo analgesia, electrical stimulation of LOFC in rats diminishes pain behavior in response to painful stimulation (Zhang et al., 1997) and has been implicated in some aspect of pain processing in nearly a dozen imaging studies (see Petrovic et al., 2000, Table 2).

In the context of placebo effects on pain, we propose that the thoughts, beliefs, and expectations associated with taking a placebo, such as thoughts about the expected reduction in pain unpleasantness, lead to increased activation in RVLPFC. This activation, according to disruption theory, should to some degree inhibit the activations in $\mathrm{dACC}$ associated with pain unpleasantness, which should bring about symptom improvements and diminished experience of pain. In other words, increased activity in RVLPFC should be related to self-reported symptom improvement, but this relation should be mediated by changes in dACC activity, which is more proximally related to the experience of pain.

\section{Overview of the study}

Patients with irritable bowel syndrome (IBS) were scanned using PET both before and following 3 weeks of taking a placebo (or active drug) for their gastrointestinal symptoms. IBS is a common syndrome characterized by chronic, recurrent abdominal pain and discomfort (associated with altered bowel habits). It is distinguished from inflammatory bowel disease by the lack of any detectable organic manifestation in the gut (Drossman et al., 2000). This has led researchers to posit that IBS symptoms may be a consequence of a hyperactive neural response to normal visceral activity (Mayer et al., 2001). One benefit of recruiting IBS patients for a study examining placebo effects is that they respond to placebos at a high rate (Spiller, 1999) relative to the range of placebo response rates found in other domains and clinical populations (Beecher, 1955; Brown et al., 1992; Kissel and Barrucand, 1964).

On scan day 1 ('pre-placebo'), patients were scanned both at rest and during controlled rectal stimulation, known to produce physical discomfort similar to IBS symptoms (Mertz et al., 1995). Patients were then given pharmacologically inactive pills to take daily for the next 3 weeks, and were told that the pill might diminish their IBS symptoms. Patients kept a symptom diary starting a week before scan day 1 and continuing throughout the 3 weeks of placebo administration. On scan day 2 ('post-placebo'), after having taken the placebo for 3 weeks, patients were again scanned multiple times at rest and during rectal stimulation.

We hypothesized that the $\mathrm{dACC}$ response to rectal stimulation would be diminished post-placebo relative to pre-placebo, but only to the extent that a placebo response occurred, operationalized as reported symptom improvements. Habituation to the experimental paradigm may account for general drops in dACC activity across the time span; however, this should not relate to the changes in self-reported symptoms. We also hypothesized that the RVLPFC response would be greater post-placebo relative to pre-placebo as a function of symptom improvement. Finally, we predicted that the relation between RVLPFC and symptom improvement would be mediated by changes in dACC, which would suggest that RVLPFC has its impact on symptoms by diminishing the reactivity of the dACC, which in turn is more directly related to symptom improvements.

\section{Methods \\ Participants}

All participants met Rome I criteria for IBS (Thompson et al., 1989) and were clinically and endoscopically without inflammatory or other structural intestinal disease. Potential participants were also excluded if they suffered any major psychiatric disorder, including clinical anxiety or depression, or scored above 63 on any two of the nine subscales or global symptom indices on the SCL90R (Derogatis and Lazarus, 1994). Patients discontinued peripherally acting treatments for IBS at least 7 days before beginning the screening phase, and centrally acting drugs at least 30 days before the first screening visit.

Of the 82 patients who were screened, 52 nonconstipated, righthanded IBS patients were enrolled in the study. Twenty-three of these patients were randomly assigned to receive placebos (the other patients were assigned to an active drug condition; data reported in Berman et al., 2002) and completed an initial PET session and were eligible for inclusion in subsequent analyses (13 women; mean age 40.7 years, range $25-60$ years). Six patients did not return for a second PET session during week 4 of the study and two additional patients did not complete a sufficient number of symptom diary entries to be included in the analyses. Thus, 15 patients in the placebo group successfully participated in the study to completion; however, one patient's PET data was corrupted and was thus excluded from these analyses. No significant differences were identified between patients who completed the study (six males, eight females) and those who did not.

\section{Procedure}

\section{Initial measures}

At the start of a 1-week screening period, patients arrived at the research unit of the UCLA Neuroenteric Disease Program, where they received a daily symptom diary to complete each evening for the duration of the study. After the screening period, patients returned to the hospital, changed into a hospital gown, then completed a battery of health and symptom-related questionnaires. Visual analogue scales (VAS) were also used to measure IBS symptom intensity and unpleasantness from the past 6 months and $24 \mathrm{~h}$ as well as several dimensions of the patient's present mood (Gracely et al., 1978).

\section{Patient preparation}

After completing the questionnaires, patients were given an enema and fitted with skin conductance and EKG electrodes, then instructed to rest quietly for $15 \mathrm{~min}$ while baseline physiological measurements were collected. Separate balloons, inflated by a computer-driven pump (barostat), were inserted into the sigmoid colon and rectum using a flexible sigmoidoscopy. For a detailed account of this procedure, see Munakata et al. (1997). Following 
insertion, patients rested in a supine position for at least $30 \mathrm{~min}$ before transfer to the PET Center.

Once at the PET Center, patients received an intravenous line. After resting quietly for $20 \mathrm{~min}$, they were fitted with headphones that delivered standardized recorded instructions and reduced extraneous noise. Patients were then placed in the PET scanner. Eyes were closed during all scans.

\section{Visceral distension}

On scan day 1 (pre-placebo), participants were given initial instructions that they would receive two rectal balloon inflations, with the first being unperceivable (baseline \#1) and the second feeling like stool or discomfort (rectal inflation \#1). During rectal inflation, the balloon was inflated to a mildly uncomfortable pressure of $45 \mathrm{~mm} \mathrm{Hg}$ for $60 \mathrm{~s}$. Patients were then informed that for the next $2 \mathrm{~min}$, they would be receiving a rectal stimulus that was significantly more intense than the preceding inflation (sham \#1). Although patients were led to anticipate a more painful rectal stimulation during this time, no inflation was applied. Another set of instructions then informed patients that the balloon in their sigmoid colon would be inflated and deflated rapidly for the next $15 \mathrm{~min}$ (noxious sigmoid inflation; one per minute at $60 \mathrm{~mm} \mathrm{Hg}$ ). Participants were told that during this time, they may feel pressure or moderate discomfort, and that if the sensation became unbearable, they should inform the experimenter who would terminate the inflation. ${ }^{3}$ The same series of rectal inflations was then repeated (baseline \#2, rectal inflation \#2, sham \#2). PET images were acquired during all baseline, rectal inflation, and sham inflation conditions, resulting in a total of six scans.

After 3 weeks of placebo treatment, patients returned for a second PET session (scan day 2, post-placebo). All procedures described above were repeated. For the purpose of the current investigation, only the rectal inflations and baseline scans on each scan day were analyzed.

\section{Symptom diary}

Symptom improvement was measured in terms of daily symptom diary response. In a daily diary, patients answered six questions about their symptoms on a scale of 0 (absent) to 10 (unbearable). Three of the diary items measuring the day's frequency, severity, and duration of abdominal pain or discomfort were used to calculate percent symptom improvement from week 1 (pre-placebo) to week 4 (post-placebo), using the following equation (negative scores indicate improvement):

$\underline{(\text { week } 4 \text { : frequency } \times \text { severity } \times \text { duration })-(\text { week } 1 \text { : frequency } \times \text { severity } \times \text { duration })}$ (week 1: frequency $\times$ severity $\times$ duration)

\section{Image acquisition and analysis}

PET was performed by dynamic imaging with a Siemens/CTI 953 tomograph (Siemens-Computer Technology, Inc., Knoxville, $\mathrm{TN})$, collecting 31 contiguous data planes corresponding to an

\footnotetext{
3 The sensitization effect of the sigmoid inflations was relevant to the drug findings reported in Berman et al. (2002); however, they are not relevant to the placebo hypotheses being tested here and as this time period was not scanned due to limitations in the number of PET scans that can be obtained per session; we will not discuss the sigmoid inflations further.
}

axial depth of $3.375 \mathrm{~mm}$ each, in a $128 \times 128$ image matrix. Emission scans were reconstructed from projection data with calculated attenuation correction. After administration of $25 \mathrm{mCi}$ ${ }^{15} \mathrm{O}$-water in a $10 \mathrm{ml}$ bolus injection of saline, PET data were obtained during four 30-s frames, and summed for frames 2-4, which followed entry of tracer into the brain.

Imaging data analysis was carried out using statistical parametric mapping (SPM'99; Wellcome Department of Cognitive Neurology, Institute of Neurology, London, UK). Images for each subject were first realigned to their initial image to correct for head motion, then normalized into a standard stereotactic space as defined by the Montreal Neurological Institute, and smoothed with a 12-mm Gaussian kernel to increase signal-to-noise ratio. Comparisons were calculated using a significance level of $P<0.005$, uncorrected for multiple comparisons combined with an extent threshold of 20 voxels - corresponding to a per-voxel, false-positive probability of less than 0.000001 (Forman et al., 1995). This method of handling multiple comparisons has been used in numerous papers (Eisenberger et al., 2003; Lieberman et al., in press; Poldrack et al., 2001; Wagner et al., 2001). Planned comparisons were computed as linear contrasts for individual participants. The resulting contrast images were then used in random effects analyses at the group level.

The neural response to visceral stimulation (i.e., discomfort or pain) on an individual scan day was computed as (rectal inflation scan \#1 + rectal inflation scan \#2) - (baseline scan \#1 + baseline scan \#2). The differential response to visceral stimulation across the two scan days was computed as [pre-placebo (rectal inflation scan $\# 1+$ rectal inflation scan \#2) - post-placebo (rectal inflation scan \#1 + rectal inflation scan \#2)].

Functional connectivity analyses were also performed (see Lieberman et al., in press; Poldrack et al., 2001) by obtaining signal intensity differences from pre-placebo to post-placebo in response to visceral stimulation in dACC for each participant. These values were then entered as regressors into a 'simple regression' analysis in SPM'99 to determine if between-subjects variation in RVLPFC activity was negatively related to betweensubjects variation in dACC activity. This analysis assesses whether individuals producing the largest decrease in dACC from pre- to post-placebo also produce the largest increase in RVLPFC from pre- to post-placebo. Similarly, symptom improvement scores were entered as regressors into a simple regression analysis to determine if between-subjects variation in symptom improvement was related to between-subjects variation in dACC and RVLPFC activity.

\section{Results}

Question 1: Was there a placebo response?

To check for significant symptom improvement, mean symptom levels from pre-treatment (week 1) and post-treatment (week 4) were computed from daily diary ratings of frequency, severity, and duration of symptoms. Symptom improvement was seen in $71 \%$ of the participants, with an average improvement of $8.5 \%$, $t(12)=2.21, P<0.05 .^{4}$ This change is in the range of large effect sizes $(r=0.54)$ according to Cohen and Cohen (1983).

${ }^{4}$ A group of patients that received the active agent Alosetron (discussed in Berman et al., 2002) showed a similar level of symptom improvement $(8.4 \%)$. 
Question 2: Was the dACC less active in response to visceral stimulation post-placebo (scan day 2) than pre-placebo (scan day 1)?

As seen in Table 1, the dACC was active both pre- and postplacebo. Though the post-placebo dACC $Z$-score is lower than the pre-placebo dACC $Z$-score, the difference is not statistically significant. This was not entirely unexpected, as it was hypothesized that the dACC activity would change more as a function of reported symptom improvement, rather than as a function of the passage of time from scan day 1 to scan day 2 or habituation to the procedure. Anterior insula was also significantly and similarly active on both scan days, consistent with the insula's role in the sensory response to visceral pain (Peyron et al., 1999).

Question 3: Were dACC changes in activity from pre-placebo to post-placebo negatively correlated with symptom improvement?

Percent symptom improvement from pre-placebo to post-placebo, as measured by the daily symptom diary, was entered as a regressor into a statistical contrast comparing the pain response on scan day 1 to the pain response on scan day 2. We looked for dACC voxels whose activity was negatively related to symptom improvement by examining a spherical region of interest (ROI) centered at $x$ $=-2, y=21, z=28$ with a $20-\mathrm{mm}$ radius (see Table $1 \mathrm{a}$ ). A cluster within this radius (see Fig. 1A) centered at $x=8, y=19, z=34$, was negatively correlated with symptom improvement $(r=-0.69$, $P<0.005)$, indicating that a greater reduction in $\mathrm{dACC}$ response to visceral stimulation in this region from pre- to post-placebo was associated with greater self-reported symptom improvement.

Question 4: Was the RVLPFC more active in response to visceral stimulation post-placebo than pre-placebo?

There was no RVLPFC activation on scan day 1 (even at $P<$ 0.05 , a lenient threshold for PET analyses); however, as seen in Table 1a, there was left-hemisphere VLPFC activation on scan day 1. On scan day 2, this pattern reversed (see Table 1b) with significant RVLPFC activity but no left-hemisphere VLPFC acti-

Table 1

Neural correlates of pain response pre-placebo (scan day 1) and postplacebo (scan day 2) 22 days later ( $P<0.005$, extent threshold of 10 voxels)

\begin{tabular}{|c|c|c|c|c|c|c|c|}
\hline \multirow{2}{*}{\multicolumn{2}{|c|}{$\begin{array}{l}\text { Region } \\
\text { (a) Scan day } 1 \text { (pain }>\text { baseline) }\end{array}$}} & & \multicolumn{3}{|c|}{$\begin{array}{l}\text { Talaraich } \\
\text { coordinate }\end{array}$} & \multirow[t]{2}{*}{ Voxels } & \multirow[t]{2}{*}{$\begin{array}{l}Z \\
\text { scor }\end{array}$} \\
\hline & & & & & & & \\
\hline Anterior cingulate & 24 & B & -2 & 21 & 28 & 113 & 3.59 \\
\hline \multirow[t]{2}{*}{ Insula } & & $\mathrm{L}$ & -36 & 6 & 2 & 150 & 3.47 \\
\hline & & $\mathrm{R}$ & 38 & 10 & -4 & 366 & 4.35 \\
\hline Amygdala & & $\mathrm{L}$ & -20 & 2 & -8 & 125 & 3.39 \\
\hline VLPFC & 47 & $\mathrm{~L}$ & -52 & 37 & -7 & 83 & 3.09 \\
\hline \multirow[t]{2}{*}{ DLPFC } & 44 & $\mathrm{~L}$ & -65 & 11 & 16 & 335 & 4.60 \\
\hline & 45 & $\mathrm{~L}$ & -38 & 38 & 20 & 197 & 3.44 \\
\hline \multicolumn{8}{|c|}{ (b) Scan day 2 (pain > baseline) } \\
\hline \multirow[t]{2}{*}{ Anterior cingulate } & 24 & $\mathrm{~B}$ & 0 & 20 & 20 & 362 & 2.79 \\
\hline & & $\mathrm{R}$ & 2 & 2 & 33 & 268 & 2.84 \\
\hline \multirow[t]{2}{*}{ Insula } & & $\mathrm{L}$ & -32 & 10 & 3 & 308 & 3.63 \\
\hline & & $\mathrm{R}$ & 36 & 12 & 0 & 179 & 3.27 \\
\hline VLPFC & 47 & $\mathrm{R}$ & 44 & 37 & -2 & 126 & 2.93 \\
\hline
\end{tabular}

All coordinates were converted from MNI to Talaraich space.

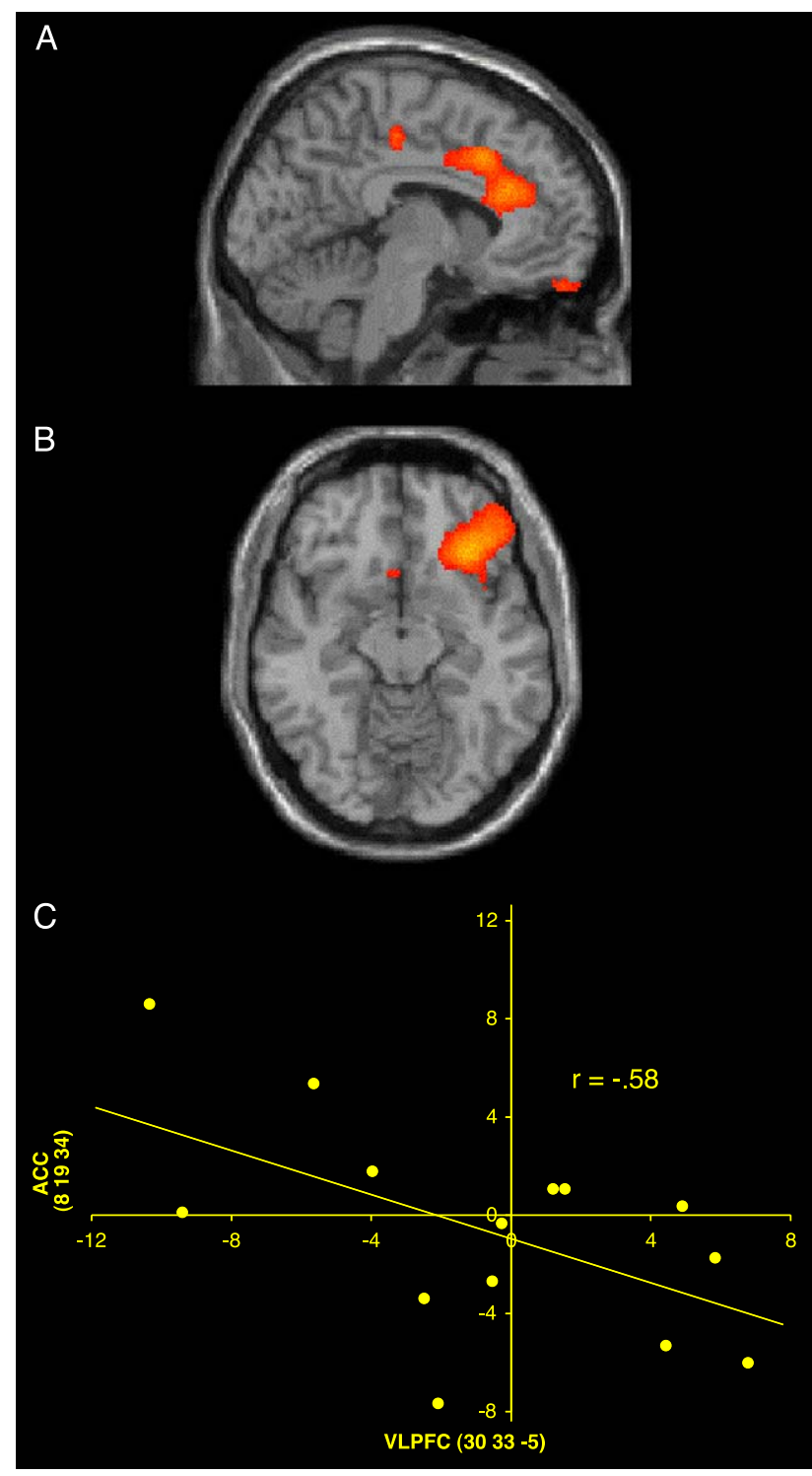

Fig. 1. (A) Dorsal anterior cingulate region that correlated negatively and (B) right ventrolateral prefrontal region that correlated positively with symptom improvement. (C) A scatterplot of relative activations showing the negative correlation between the dorsal anterior cingulate and right ventrolateral prefrontal cortex. Each point is a single participant's change in activity, in relative units, from scan day 1 to scan day 2 .

vation. Comparisons across the two scan days were not significant in either region of VLPFC. As with the dACC activity, we expected that RVLPFC changes from pre- to post-placebo would be a function of symptom improvement rather than the passage of time or habituation, so this result was not unexpected.

Question 5: Were RVLPFC changes in activity from pre- to post-placebo positively correlated with symptom improvement?

Percent symptom improvement from pre- to post-placebo was entered as a regressor into a statistical contrast comparing the pain response on scan day 1 to the pain response on scan day 2. We looked for RVLPFC voxels whose activity were positively related to symptom improvement by examining a spherical region of 
interest (ROI) centered at $x=44, y=37, z=-2$ with a $20-\mathrm{mm}$ radius (see Table 1b). A cluster within this radius (see Fig. 1B) centered at $x=30, y=33, z=-5$, was positively correlated with symptom improvement $(r=0.71, P<0.005)$, indicating that greater RVLPFC increases from pre- to post-placebo were associated with greater self-reported improvement.

Question 6: Were the changes in RVLPFC and dACC activity from pre- to post-placebo negatively correlated with one another?

A functional connectivity analysis was computed on the dACC activation at $x=8, y=19, z=34$ and the RVLPFC activation at $x=$ $30, y=33, z=-5$ identified in the analyses described in questions $\# 3$ and \#5 above. The activations in these two regions were found to be negatively correlated with one another $(r=-.55, P<0.05)$ such that participants who had a larger increase in RVLPFC activity from scan day 1 to scan day 2 had a larger decrease in dACC activity from scan day 1 to scan day 2 (Fig. 1C).

Question 7: Was the positive correlation between RVLPFC activity and symptom improvement mediated by dACC activity?

We hypothesized that the positive relationship between RVLPFC changes from scan day 1 to scan day 2 and symptom improvement would be mediated by changes in dACC activity. That is, RVLPFC was expected to have its effect on symptom improvement by reducing dACC activity, which would lead to symptom improvement. Because each of the zero-order correlations between the three factors were significant, the prerequisite for running mediational analyses was fulfilled (Baron and Kenny, 1986). The analysis (see Fig. 2) indicated that dACC activity did mediate the direct path from RVLPFC to symptom improvement (Sobel test, $Z=1.98, P<0.05$ ). After controlling for dACC activity, the remaining path from RVLPFC to symptom improvement was no longer significant $(P>0.13)$ and the amount of variance accounted for in symptom improvement dropped $60 \%$ from an $r^{2}=0.50$ to $r^{2}=0.18$.

\section{Habituation and other effects}

We noted earlier that changes in dACC from pre- to postplacebo that were related to self-reported symptom improvement are not likely to be the result of habituation and other effects. We performed an analysis of covariance (ANCOVA), removing symptom improvement as a nuisance variable, to determine if any activity in AACC could be attributed to habituation and other

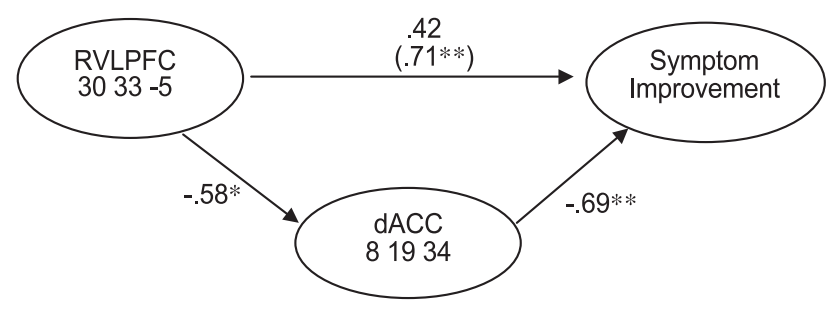

Fig. 2. The mediating role of dorsal anterior cingulate (dACC) between right ventrolateral prefrontal (RVLPFC) and symptom improvement. Numbers are standardized coefficients. The number in parentheses indicates the direct effect of RVLPFC on symptom improvement, before the inclusion of the dACC mediator in the regression. $* P<0.05, * * P<0.005$. effects once placebo effects were removed. At our strict significance and extent threshold levels, no activations were found in dACC or elsewhere in the brain. When we lowered our extent threshold to a more lenient criteria of 10 voxels, we did find a region of left dACC $(x=-10, y=28, z=44)$ that was significantly less active $(Z=2.68, P=0.004)$, suggesting that nonplacebo effects were also altering the dACC response over time. This region of activity did not, however, overlap with the region in right dACC associated with the symptom improvements.

\section{Discussion}

This is the first neuroimaging study to investigate the functional contribution of RVLPFC in the placebo response. Petrovic et al. (2002) had previously observed that RVLPFC was activated during placebo analgesia but not during opioid analgesia. In the current study, we observed a strong positive correlation between pre- to post-placebo increases in RVLPFC and subjective reports of symptom improvement. Mediational analyses indicated that this relationship was mediated by $\mathrm{dACC}$ such that increased activity in RVLPFC was associated with decreased activity in dACC, which in turn was negatively correlated with symptom improvement. The regions of RVLPFC and dACC involved in this mediation process are close to those found by Petrovic et al. (2002) ( $x=30, y=30, z=-6$ in RVLPFC) and Rainville et al. (1997) $(x=3, y=20, z=30$ in dACC). They are also similar to the regions observed in a study of social pain, which also demonstrated the same mediational pattern such that right ventral PFC effects on social pain were mediated by dACC changes (Eisenberger et al., 2003).

These results suggest that placebos may operate, in part, by increasing thoughts about the affective aspects of the pain (i.e., "I believe I am going to be less bothered by pain now") associated with increased activity in RVLPFC. As predicted by disruption theory, any activation of RVLPFC is expected to inhibit dACC activity, which in turn should diminish pain unpleasantness. Such a process is consistent with previous research indicating that expectations play an important role in placebo effects (Montgomery and Kirsch, 1997), as expectations typically involve affective thoughts about current and future pain experiences. It remains an open question, however, whether expectation and belief processes have their effect on RVLPFC during the inflations themselves or if RVLPFC's response to pain was altered as a result of belief processes during the 3 weeks of placebo administration between scanning sessions. Disruption theory is most consistent with belief processes having their effect during the inflations. Because thought processes during the inflations were not assessed, we cannot rule out the possibility that processes before the second scan were driving the RVLPFC activations.

Additionally, because we did not assess the extent to which participants engaged in affective thought processes related to their beliefs and expectations, we cannot say whether RVLPFC activity was a result of these thought processes or some other nonaffective processes. Obtaining retrospective self-report of affective thought would have proved problematic as such self-reports are often in error (Nisbett and Wilson, 1977). Moreover, if participants knew that such self-report measures were going to be taken, it could well have altered the content of the thought processes that did occur (Wegner, 1989). 
While disruption theory may provide a neurally plausible route by which belief and expectations might produce placebo effects, we do not mean to suggest that this is the only route by which placebo effects occur in the brain. Numerous studies have also shown that placebo effects can result from conditioning procedures. In these studies, participants are usually exposed to an active agent that is then replaced with a placebo (e.g., Amanzio and Benedetti, 1999). In our study, participants were not exposed to an active agent at any time, and thus our study does not speak to this mechanism by which placebo effects also occur. Thus, while other pathways to placebo effects may exist, this paper has presented the first evidence of a multistep neural path from placebo to brain to subjective report.

One concern in the current study is that we did not have a natural history group that underwent both scanning procedures without receiving placebos. Because of the prohibitive expense of scanning, we relied on scanning the placebo group both before and after taking the placebos for 3 weeks. One might, at first glance, think that any changes from pre- to post-placebo might be attributable to the passing of time or habituation effects, but if this were the case, the reductions in dACC from pre- to postplacebo should be unrelated to changes in symptomology in the intervening 3 weeks. placebo In other words, individuals might have become habituated to the scanner procedure from scan day 1 to scan day 2, but this should lead to a main effect decrease in dACC activity over time without any changes related to the magnitude of self-reported symptom change during the intervening 3 weeks. We found no main effect decrease in dACC activity that a habituation account would predict. Rather, the variance in dACC from pre- to post-placebo was better accounted for by the change in self-reported symptomology. Habituation effects were also present, but these effects were associated with a different region of dACC from the placebo-related effects.

The only other way habituation effects could provide an alternative account for our data is if the initial scanning procedure caused the participants' daily IBS symptoms to abate to the same extent that it caused participants to habituate neurally to the scanner procedure. In this scenario, participants who habituated more to the scanner procedure would also have fewer self-reported IBS symptoms in the intervening 3 weeks, thus artificially producing the correlation we observed between ACC change and symptom change across the two time points. Several of the authors of this paper (Naliboff et al., 2001) have conducted a longitudinal PET imaging study of IBS patients that suggests this possibility is unlikely. In that study, which included neither a placebo nor an active drug component, IBS patients participated in six visceral stimulation protocols over 1 year, the first and last including PET imaging. Naliboff et al. found some habituation in the ACC to the pain induction and scanner procedure; however, symptom reports remained stable over the course of the year. In other words, habituation to the scanner procedure did not lead to diminished symptoms outside the scanner. This suggests that although it is likely that there were some ACC changes in our study that can be attributed to habituation, those changes would not have covaried with the symptom reports. Consequently, the ACC changes that did covary with symptom reports were likely the result of the placebo induction.

Finally, it could be argued that spontaneous remission could have also produced the symptom improvements seen from scan day 1 to scan day 2 . In this case, the absence of a natural history group would leave placebo effects confounded with spontaneous symptom improvements. The Naliboff et al. (2001) longitudinal study found that IBS symptoms were quite stable over the course of six assessments in a 1-year period. Thus, it seems unlikely that spontaneous recovery would account for the results of this study.

While a natural history group would have provided additional controls for spontaneous changes in symptom levels, we note that several recent placebo pain studies that have used natural history groups (Amanzio and Benedetti, 1999; Benedetti et al., 2003; Vase et al., 2003) have not found significant variation over time in the natural history group. One of these studies (Vase et al., 2003) also focused on IBS patients. The lack of time effects in natural history groups suggests that the inferential cost of leaving them out of our study is a reasonable trade-off for the cost of scanning a control group twice.

In summary, we examined the placebo effect for pain in a population of IBS patients. These individuals reported significant symptom improvements over the course of a 3-week placebo regimen. The dACC reactivity to physical discomfort was reduced from pre- to post-placebo to the extent that participants reported symptom improvement. Conversely, the RVLPFC response to physical discomfort increased from pre- to post-placebo to the extent that participants reported symptom improvement. The changes in activity in these two brain regions were negatively correlated and a mediational analysis revealed that changes in dACC mediated the relationship between RVLPFC and symptom changes. This suggests that whereas dACC activity may be most directly associated with symptom improvement in response to the placebo administration, the RVLPFC may produce the dACC changes and serve a more central role in placebo effects. Given that placebo effects depend on the belief that the placebo will improve some condition that is causing distress, and given that RVLPFC has been associated with affective thought and the inhibition of affect, thought, and behavior, RVLPFC may serve to convert placebo-related beliefs into the neural, behavioral, and experiential placebo effects that have fascinated humans throughout time.

\section{Acknowledgments}

We thank Leah Fitzgerald, N.P, Ph.D., and Jean Stains, R.N., for invaluable assistance in executing the study; and Molly Crockett, Naomi Eisenberger, Sarah Master, Jennifer Pfeifer, Ajay Satpute, and Robert Spunt for comments on an earlier draft. This work was supported in parts by grants from National Institute of Diabetes and Digestive and Kidney Diseases (DK48351 and DK64539) to E.A.M., from the National Institute of Mental Health to B.N. (R01NR07768), M.D.L (R21MH66709-01), and J.M.J. (National Research Service Award through the UCLA Health Psychology Training Grant) and by funds from GlaxoSmithKline. We would also like to thank the UCLA Brain Mapping Center for computing resources supporting this work.

\section{References}

Amanzio, M., Benedetti, F., 1999. Neuropharmacological dissection of placebo analgesia: expectation-activated opioid systems versus conditioning-activated specific subsystems. J. Neurosci. 19, 484-494.

Aron, A.R., Fletcher, P.C., Bullmore, E.T., Sahakian, B.J., Robbins, T.W., 2003. Stop-signal inhibition disrupted by damage to right inferior frontal gyrus in humans. Nat. Neurosci. 6, 115-116. 
Baron, R.M., Kenny, D.A., 1986. The moderator-mediator variable distinction in social psychological research: conceptual, strategic, and statistical considerations. J. Pers. Soc. Psychol. 51, 1173-1182.

Beecher, H.K., 1955. The powerful placebo. J. Am. Med. Assoc. 15, $1602-1606$.

Benedetti, F., Pollo, A., Lopiano, L., Lanotte, M., Vighetti, S., Rainero, I., 2003. Conscious expectation and unconscious conditioning in analgesic, motor, and hormonal placebo/nocebo responses. J. Neurosci. 23, $4315-4323$.

Berman, S.M., Chang, L., Suyenobu, B., Derbyshire, S.W., Stains, J., Fitzgerald, L., Mandelkern, M., Hamm, L., Vogt, B., Naliboff, B.D., Mayer, E.A., 2002. Condition-specific deactivation of brain regions by 5-HT3 receptor antagonist alosetron. Gastroenterology 123, 969-977.

Brown, W.A., Johnson, M.F., Chen, M.G., 1992. Clinical features of depressed patients who do and do not improve with placebo. Psychiatry Res. 41, 203-214.

Carmichael, S.T., Price, J.L., 1995. Limbic connections of the orbital and medial prefrontal cortex in macaque monkeys. J. Comp. Neurol. 363, $615-641$.

Cavada, C., Company, T., Tejedor, J., Cruz-Rizzolo, R.J., Reinoso-Suarez, F., 2000. The anatomical connections of the Macaque monkey orbitofrontal cortex. A review. Cereb. Cortex 10, 220-242.

Coghill, R.C., Sang, C.N., Maisog, J.M., Iardarola, M.J., 1999. Pain intensity processing within the human brain: a bilateral, distributed mechanism. J. Neurophysiol. 82, 1934-1943.

Cohen, J., Cohen, P., 1983. Applied Multiple Regression/Correlation Analysis for the Behavioral Sciences, second ed. Erlbaum, Hillsdale, NJ.

Crosson, B., Radanovich, K., Sadek, J.R., Gokcay, D., Bauer, R.M., Fischler, I.S., et al., 1999. Left-hemisphere processing of emotional connotation during word generation. NeuroReport 10, 2449-2455.

Cunningham, W.A., Johnson, M.K., Gatenby, J.C., Gore, J.C., Banaji, M.R., 2003. Neural components of social evaluation. J. Pers. Soc. Psychol. 85, 639-649.

de la Fuente-Fernandez, R., Ruth, T.J., Sossi, V., Schulzer, M., Calne, D.B., Stoessl, A.J., 2001. Expectation and dopamine release: mechanism of the placebo effect in Parkinson's disease. Science 293, 1164-1166.

Derogatis, L.R., Lazarus, L., 1994. SCL-90-R, Brief Symptom Inventory, and matching clinical rating scales. In: Maruish, M. (Ed.), The Use of Psychological Testing for Treatment Planning and Outcome Assessment. Erlbuam, Hillsdale, NJ, pp. 217-248.

Drossman, D.A., Corazziari, E., Talley, N.J., Thompson, W.G., Whitehead, W.E., 2000. ROME: II. The Functional Gastrointestinal Disorders. Diagnosis, Pathophysiology and Treatment: A Multinational Consensus Consensus. Degnon Associates, McLean, VA.

Eisenberg, N.I., Lieberman, M.D., Williams, K.D., 2003. Does rejection hurt? An fMRI study of social exclusion. Science 302, 290-292.

Eisenberg, N.I., Lieberman, M.D. (in press). Why it hurts to be left out: the neurocognitive overlap between physical and social pain. In: Williams, K.D., Forgas, J.P., von Hippel, W. (Eds.), The Social Outcast: Ostracism, Social Exclusion, Rejection, and Bullying. New York: Cambridge University Press.

Eugene, F., Levesque, J., Mensour, B., Leroux, J., Beaudoin, G., Bourgouin, P., Beauregard, M., 2003. The impact of individual differences on the neural circuitry underlying sadness. NeuroImage 19, 354-364.

Fiske, S.T., Taylor, S.E., 1991. Social Cognition. McGraw-Hill, New York. Foltz, E.L., Lowell, E.W., 1962. Pain "relief" by frontal cingulomotomy. J. Neurophysiol. 80, 89-100.

Forman, S.D., Cohen, J.D., Fizgerald, M., Eddy, W.F., Mintun, M.A., Noll, D.C., 1995. Improved assessment of significant activation in functional magnetic resonance imaging (fMRI): use of cluster-size threshold. Magn. Reson. Med. 33, 636-647.

Garavan, H., Ross, T.J., Stein, E.A., 1999. Right hemispheric dominance of inhibitory control: an event-related functional MRI study. Proc. Natl. Acad. Sci. U. S. A. 96, 8301-8306.

Gorno-Tempini, M.L., Pradelli, S., Serafini, M., Pagnoni, G., Baraldi, P., Porro, C., Nicoletti, R., Umita, C., Nichelli, P., 2001. Explicit and incidental facial expression processing. An fMRI study. NeuroImage $14,465-473$.

Gottfried, J.A., Deichmann, R., Winston, J.S., Dolan, R.J., 2002. Functional heterogeneity in human olfactory cortex: an event-related functional magnetic resonance imaging study. J. Neurosci. 22, 10819-10828.

Gracely, R.H., McGrath, P., Dubner, R., 1978. Ratio scales of sensory and affective verbal pain descriptors. Pain 5, 5-18.

Gur, R.C., Schroeder, L., Turner, T., McGrath, C., Chan, R.M., Turetsky, B.I., Alsop, D., Maldjian, J., Gur, R.E., 2002. Brain activation during facial emotion processing. NeuroImage 16, 651-662.

Hariri, A.R., Bookheimer, S.Y., Mazziotta, J.C., 2000. Modulating emotional response: effects of a neocortical network on the limbic system. NeuroReport 11, 43-48.

Hrobjartsson, A., Gotzsche, P.C., 2001. Is the placebo powerless? An analysis of clinical trials comparing placebo with no treatment. N. Engl. J. Med. 344, 1594-1602.

Iversen, S.D., Mishkin, M., 1970. Perseverative interference in monkeys following selective lesions of the inferior prefrontal convexity. Exp. Brain Res. 11, 376-386.

Kissel, P., Barrucand, D., 1964. Placebo and Placebo Effect in Medicine. Masson, Paris, France.

Konishi, S., Nakajima, K., Uchida, I., Kikyo, H., Kameyama, M., Miyashita, Y., 1999. Common inhibitory mechanism in human inferior prefrontal cortex revealed by event-related functional MRI. Brain 122, 981-999.

LeDoux, J.E., 1996. The Emotional Brain: The Mysterious Underpinnings of Emotional Life. Simon \& Schuster, New York

Leuchter, A.F., Cook, I.A., Witte, E.A., Morgan, M., Abrams, M., 2002. Changes in brain function of depressed subjects during treatment with placebo. Am. J. Psychiatry 159, 122-129.

Lieberman, M.D., 2003. Reflective and reflexive judgment processes: a social cognitive neuroscience approach. In: Forgas, J.P., Williams, K.R., von Hippel, W. (Eds.), Social Judgments: Explicit and Implicit Processes. Cambridge Univ. Press, New York, pp. 44-67.

Lieberman, M.D., Hariri, A., Jarcho, J.M., Bookheimer, S.Y., 2003. Two captains, one ship: a social cognitive neuroscience approach to disrupting automatic affective processes. Unpublished manuscript.

Lieberman, M.D., Chang, G.Y., Chiao, J., Bookheimer, S.Y., Knowlton, B.J., in press. An event-related fMRI study of artificial grammar learning in a balanced chunk strength design. J. Cogn. Neurosci.

Markowitsch, H.J., Vandekerckhove, M.M.P., Lanfermann, H., Russ, M.O., 2003. Engagement of lateral and medial prefrontal areas in the ecphory of sad and happy autobiographical memories. Cortex 39, 1-23.

Mayberg, H.S., Silva, J.A., Brannan, S.K., Tekell, J.L., Mahurin, R.K., McGinnis, S., et al., 2002. The functional neuroanatomy of the placebo effect. Am. J. Psychiatry 159, 728-737.

Mayer, E.A., Naliboff, B.D., Chang, L., Coutinho, S.V., 2001. Stress and irritable bowel syndrome. Am. J. Physiol.: Gastrointest. Liver Physiol. 280, 519-524.

Melzack, R., Casey, K.L., 1968. Sensory, motivational, and central control determinants of pain. In: Kenshalo, D.R. (Ed.), The Skin Senses. CC Thomas, Springfield, IL, pp. 423-439.

Mertz, J.R., Naliboff, B., Munakata, J., Niazi, N., Mayer, E.A., 1995. Altered rectal perception is a biological marker of patients with irritable bowel syndrome. Gastroenterology 109, 40-52.

Monchi, O., Petrides, M., Petre, V., Worsley, K., Dagher, A., 2001. Wisconsin card sorting revisited: distinct neural circuits participating in different stages of the task identified by event-related functional magnetic resonance imaging. J. Neurosci. 21, 7733-7741.

Montgomery, G.H., Kirsch, I., 1997. Classical conditioning and placebo effect. Pain 72, 107-113.

Munakata, J., Naliboff, B., Harraf, F., Kodner, A., Lembo, T., Chang, L., Silverman, D.H., Mayer, E.A., 1997. Repetitive sigmoid stimulation induces rectal hyperalgesia in patients with irritable bowel syndrome. Gastroenterology 113, 1054.

Nakamura, K., Kawashima, R., Ito, K., Sugiura, M., Kato, T., Nakamura, A., Hatano, K., Nagumo, S., Kubota, K., Fukuda, H., Kojima, S., 
1999. Activation of the right inferior frontal cortex during assessment of facial emotion. J. Neurophysiol. 82, 1610-1614.

Naliboff, B., Derbyshire, S.W.G., Berman, S., Mandelkern, M., Chang, L., Stains, J., Mayer, E.A., 2001. Assessing the stability of brain activation to visceral stimulation in IBS. Gastroenterology 120, A133.

Narumoto, J., Yamada, H., Iidaka, T., Sadato, N., Fukui, K., Itoh, H., Yonekura, Y., 2000. Brain regions involved in verbal or non-verbal aspects of facial emotion recognition. NeuroReport 11, 2571-2576.

Nisbett, R.E., Wilson, T.D., 1977. Telling more than we can know: verbal reports on mental processes. Psychol. Rev. 84, 231-259.

Ongur, D., Price, J.L., 2000. The organization of networks within the orbital and medial prefrontal cortex of rats, monkeys and humans. Cereb. Cortex 10, 206-219.

Petrovic, P., Petersson, K.M., Ghatan, P.H., Stone-Elander, S., Ingvar, M., 2000. Pain-related cerebral activation is altered by a distracting cognitive task. Pain 85, 19-30.

Petrovic, P., Kalso, E., Petersson, K.M., Ingvar, M., 2002. Placebo and opioid analgesia: imaging a shared neuronal network. Science 295 , $1737-1740$.

Peyron, R., Garcia-Larrea, L., Gregoire, M., Costes, N., Convers, P., Lavenne, F., et al., 1999. Haemodynamic brain responses to acute pain in humans: sensory and attentional networks. Brain 122, 1765-1779.

Peyron, R., Laurent, B., Garcia-Larrea, L., 2000. Functional imaging of brain responses to pain. A review and meta-analysis. Clin. Neurophysiol. 30, 263-288.

Ploner, M., Freund, H.J., Schnitzler, A., 1999. Pain affect without pain sensation in a patient with a postcentral lesion. Pain 81, 211-214.

Poldrack, R.A., Clark, J., Paré-Blagoev, E.J., Shohamy, D., Creso Moyano, J., Myers, C., Gluck, M.A., 2001. Interactive memory systems in the human brain. Nature 414, 546-550.

Price, D., 2000. Psychological and neural mechanisms of the affective dimension of pain. Science 288, 1769-1772.

Rainville, P., Duncan, G.H., Price, D.D., Carrier, B., Bushnell, M.C., 1997. Pain affect encoded in human anterior cingulate but not somatosensory cortex. Science 277, 968-971.

Schaefer, A., Collette, F., Philippot, P., Van der Linden, M., Laureys, S., Delfiore, G., Degueldre, C., Maquet, P., Luxen, A., Salmon, E., 2003. Neural correlates of disruption account of placebos 30 "hot" and "cold" emotional processing: a multilevel approach to the functional anatomy of emotion. NeuroImage 18, 938-949.

Schwarz, N., Bless, H., 1991. Happy and mindless, but sad and smart? The impact of affective states of on analytical reasoning. In: Forgas, J.P. (Ed.), Emotion and Social Judgments. Pergamon, Oxford, pp. 55-71.

Small, D.M., Zatorre, R.J., Dagher, A., Evans, A.C., Jones-Gotman, M., 2001. Changes in brain activity related to eating chocolate. From pleasure to aversion. Brain 124, 1720-1733.

Spiller, R.C., 1999. Problems and challenges in the design of irritable bowel syndrome clinical: experience from published trials. Am. J. Med. 107, 91S-97S.

Teasdale, J.D., Howard, R.J., Cox, S.G., Ha, Y., Brammer, M.J., Williams, S.C.R., Checkley, S.A., 1999. Functional MRI study of the cognitive generation of affect. Am. J. Psychiatry 156, 209-215.

Thompson, W.G., Dotevall, G., Drossman, D.A., Heaton, K.W., Kruis, W., 1989. Irritable bowel syndrome: guidelines for the diagnosis. Gastroenterol. Int. 2, 92-95.

Tolle, T.R., Kaufmann, T., Siessmeier, T., Lautenbacher, S., Berthele, A., Munz, F., et al., 1999. Region-specific encoding of sensory and affective components of pain in the human brain: a positron emission tomography correlation analysis. Ann. Neurol. 45, 40-47.

Vase, L., Robinson, M.E., Verne, G.N., Price, D.D., 2003. The contributions of suggestion, desire, and expectation to placebo effects in irritable bowel syndrome patients. An empirical investigation. Pain 105, $17-25$.

Vogt, B.A., Pandya, D.N., 1987. Cingulate cortex of the Rhesus monkey: II. Cortical afferents. J. Comp. Neurol. 262, 271-289.

Wagner, A.D., Pare-Blagoev, E.J., Clark, J., Poldrack, R.A., 2001. Recovering meaning: left prefrontal cortex guides controlled semantic retrieval. Neuron 31, 329-338.

Wegner, D.M., 1989. White Bears and Other Unwanted Thoughts. Guilford, New York, NY.

Wegner, D.M., Bargh, J.A., 1998. Control and automaticity in social life. In: Gilbert, C., Fiske, S.T., Lindzey, G. (Eds.), The Handbook of Social Psychology. Oxford Univ. Press, New York, pp. 446-496.

Zhang, Y., Tang, J., Yuan, B., Jia, H., 1997. Inhibitory effects of electrically evoked activation of ventrolateral orbital cortex on the tail-flick reflex are mediated by periaqueductal gray in rats. Pain $72,127-135$. 